\title{
Evidence of oxidative stress-induced senescence in mature, post-mature and pathological human placentas
}

\author{
Tereza Cindrova-Davies ${ }^{\mathrm{a}, *}$, Norah M.E. Fogarty ${ }^{\mathrm{a}, \mathrm{b}}$, Carolyn J.P. Jones ${ }^{\mathrm{c}}$, John Kingdom ${ }^{\mathrm{d}}$, \\ Graham J. Burton ${ }^{\text {a }}$ \\ ${ }^{\text {a }}$ Centre for Trophoblast Research, University of Cambridge, UK \\ ${ }^{\mathrm{b}}$ Francis Crick Institute, London, UK \\ ${ }^{\mathrm{c}}$ Maternal and Fetal Health Research Centre Division of Developmental Biology \& Medicine School of Medical Sciences Faculty of Biology, Medicine and Health University \\ of Manchester, Manchester Academic Health Science Centre, St Mary's Hospital Oxford Road Manchester M139 WL, UK \\ ${ }^{\mathrm{d}}$ Mount Sinai Hospital, University of Toronto, Canada
}

A R T I C L E I N F O

\section{Keywords:}

Senescence

Syncytiotrophoblast

Oxidative stress

\begin{abstract}
A B S T R A C T
Introduction: Premature ageing has been implicated in placental dysfunction. Senescence can be activated by oxidative stress, a key intermediary in the pathophysiology of pre-eclampsia. We examined senescence markers across normal gestation, and in pathological and post-mature pregnancies. Inducers of oxidative stress were used to mimic senescence changes in term explants.

Methods: Placental samples were collected with ethical approval and informed consent: first and second trimester samples from surgical terminations; term and pre-term controls, and early-onset pre-eclampsia samples from caesarean deliveries. Paraffin and EM blocks of post-mature placentas were from an archival collection. Term explants were subjected to hypoxia-reoxygenation (HR) or hydrogen peroxide $\left(\mathrm{H}_{2} \mathrm{O}_{2}\right)$.

Results: p21 was increased significantly in term homogenates compared to first and second trimester samples, and was significantly higher in PE compared to term controls. Immunostaining revealed nuclear localisation of p21 and phosphorylated histone $\gamma \mathrm{H} 2 \mathrm{AX}$ in syncytiotrophoblast, with abundant foci in pathological and postmature placentas. Abnormal nuclear appearances were observed in post-mature placentas. Sudan-Black-B staining demonstrated abundant lipofuscin, an aggregate of oxidised proteins, lipids and metals, in post-mature and pathological placentas. The percentage of nuclei positive for 8-hydroxy-2'-deoxy-guanosine, a marker of oxidised DNA/RNA, was increased in pathological placentas compared to age-matched controls. These changes could be mimicked by challenge with $\mathrm{HR}$ or $\mathrm{H}_{2} \mathrm{O}_{2}$.

Discussion: Senescence markers increase in normal placentas with gestational age, and are exaggerated in postmature and pathological cases. Oxidative stress triggers equivalent changes in explants, and may precipitate senescence in vivo. The consequent pro-inflammatory senescence-associated secretory phenotype may contribute to the pathophysiology of pre-eclampsia.
\end{abstract}

\section{Introduction}

Senescence is a critical feature of mammalian cells, and can be both beneficial and detrimental. Thus, oncogene-triggered senescence suppresses tumour growth [1], whereas loss of tissue homeostasis during ageing [2,3] contributes to atherosclerosis [4] and neurodegeneration [5]. Senescence has been implicated in cell fusion [6], and so is relevant to the syncytiotrophoblast of the human placenta. Transfection of normal and malignant non-trophoblast cells with syncytin 1 causes cellular fusion and formation of syncytia, which exhibit features of senescence. Replicative senescence is a permanent cell cycle arrest resulting from high levels of the cyclin kinase inhibitors p21/or p16. Cells remain metabolically active and adopt characteristic phenotypic changes [7]. Cell senescence can also be activated by a variety of intrinsic and extrinsic stresses, including oxidative stress, DNA damage, nucleolar stress, epigenetic stress, telomere damage, chronic mitogen signaling, and oncogene activation/inactivation. For example, reactive oxygen species activate DNA damage response (DDR) by perturbing gene transcription and DNA replication, and by inducing telomere shortening. Senescence stressors ultimately activate the p53 and/or p16 ${ }^{\text {Ink4a }}$ pathways; 553 activation inducing p 21 and cell cycle arrest via cyclin E-Cdk2, while p16 ${ }^{\text {Ink4a }}$ targets cyclin D-Cdk4 and D-Cdk6

\footnotetext{
* Corresponding author. Centre for Trophoblast Research, Physiological Laboratory, Downing Street, Cambridge CB2 3EG, UK.

E-mail address: tc269@cam.ac.uk (T. Cindrova-Davies).
} 
complexes [8]. These actions prevent inactivation of RB (retinoblastoma protein), leading to continued repression of E2F target genes required for S-phase onset. Some cells form regions of highly condensed chromatin, called senescence-associated heterochromatin foci, that sequester genes facilitating cell-cycle control. These foci contain chromatin modifications, such as activated $\mathrm{H} 2 \mathrm{AX}(\gamma \mathrm{H} 2 \mathrm{AX})$ and H3K9me, and reinforce senescence-associated growth arrest [9,10]. One emerging feature of senescent cells is inflammation. Senescent cells secrete a unique cocktail of factors, collectively known as senescence-associated secretory phenotype (SASP), which includes pro-inflammatory cytokines and chemokines, and various growth factors and proteases that together alter the tissue microenvironment [11,12]. Damaged human cells develop persistent chromatin lesions bearing hallmarks of DNA double-strand breaks, which initiate increased secretion of inflammatory cytokines. Evidence suggests that a feedback loop involving mitochondrial dysfunction and ROS production might be important in various physiologically relevant forms of cell senescence [13]. Senescent cells have distinct phenotypic features, including flattened and enlarged morphology with marked actin stress fibres, stability in culture, and increased senescence-associated $\beta$-galactosidase (SA- $\beta$-gal) activity [14]. SA- $\beta$-gal is the most reliable senescence biomarker. However, the SA- $\beta$-gal activity can be detected only in snap-frozen tissues, not in archival paraffin-embedded sections. A recent study validated the histochemical Sudan-Black-B (SBB) specific stain of lipofuscin, an aggregate of oxidised proteins, lipids and metals known to accumulate in aged tissues, as an additional reliable approach to detect senescent cells independently of sample preparation [15]. In addition, cytoplasmic chromatin fragments can pinch off from intact nuclei of primary cells during senescence. Recent evidence shows that the presence of DNA in the cytoplasm can initiate a SASP response by activating the innate immunity cytosolic DNA-sensing cyclic GMP-AMP synthase (cGAS)-STING pathway [16,17]. The enzyme cyclic GMP-AMP synthase (cGAS) acts as a first responder as it binds to cytoplasmic DNA, and catalyses production of the molecule cGAMP and triggers a proinflammatory response $[16,17]$.

Placental dysfunction is the main cause of many placental-related pathologies. Recent studies indicate that premature placental ageing might be involved in this process. Placental ageing was first described in the 1970s in animal models [18-20] when it was suggested that the rapidly proliferating placental cells start to age as the placenta reaches term. This physiological ageing has been documented in a recent study of human term placentas (39 wk), late term placentas ( $>41 \mathrm{wk})$ and placentas from unexplained stillbirth. Increased aldehyde oxidase 1 expression, increased oxidation of DNA/RNA (8-OHdG) and lipid, perinuclear location of lysosomes, and larger autophagosomes were observed in both later term and stillborn placentas, compared to placentas from term deliveries [21]. In addition, the significant increase in the rate of stillbirth commencing at 36-37 wk gestation, suggests that placental ageing might increase the risk of fetal demise [22,23]. Thus the first aim of this study was to examine evidence of senescence across normal gestation, and to evaluate senescent changes in an archival collection of post-mature placentas (delivered 7-20 after the due date).

Strong evidence exists that placental oxidative stress is a key intermediary event in the pathology of preeclampsia (PE) and intrauterine growth restriction (IUGR) [24-26], secondary to deficient conversion of the uterine spiral arteries and malperfusion [27]. Damage to DNA and proteins can result in shortened telomeres in the trophoblast in these pregnancies [28], and induce senescence. In addition, a recent microarray analysis demonstrated increased expression of $p 21$, p53, APE1 and $I L-6$ in PE and IUGR placentas [29]. Therefore, the second aim of this study was to investigate senescent changes in pathological pre-eclamptic and IUGR pregnancies, and to use inducers of oxidative stress to test whether they are capable of recapitulating senescence changes in healthy term placental explants in vitro.

\section{Methods}

Tissue collection - first, second trimester and term tissue

All material was collected with informed written patient consent. First and second trimester placental samples were collected from surgical terminations with approval of the Joint UCL/UCLH Committees on the Ethics of Human Research (05/Q0505/82). The samples were collected using a chorionic villus sampling-like (CVS) technique under ultrasound guidance from the central region of the placenta. Gestational age was estimated from the crown rump length of the fetus. Part of the sample was frozen immediately $(<2 \mathrm{~min}$ ) in liquid nitrogen (time zero, $\mathrm{T}_{0}$ ), and part was fixed in $4 \%$ paraformaldehyde (PFA) and embedded in paraffin wax for immunohistochemistry (IHC). Frozen samples of first trimester $(7-8 \mathrm{wk} ; \mathrm{N}=5)$ and second trimester $(13-17 \mathrm{wk} ; \mathrm{N}=6)$ placentas were analysed in this study. To study changes across gestation, samples were collected from uncomplicated singleton pregnancies at term $(\mathrm{N}=5 ; 39$ weeks) with approval from the Cambridge Local Ethics Committee and with informed written patient consent immediately after delivery by elective caesarean section (tissues were harvested within $10 \mathrm{~min}$ of delivery). The most common indication for caesarean section was a history of previous section. Samples were transported to the laboratory on ice for further processing or for use as explant cultures (see below).

\section{Tissue collection - post-mature placentas and their term controls}

Paraffin and EM blocks of term healthy placentas $(\mathrm{N}=5)$ and postmature placentas (delivered $7-20 \mathrm{~d}$ after due date, $\mathrm{N}=6$ ) were from an archival collection from the University of Manchester [30].

\section{Tissue collection - pathological samples and appropriate controls}

Pathological placentas and respective term and preterm controls were collected with ethical approval from the Mount Sinai Hospital, Toronto, Canada, following caesarean delivery. Samples included term controls $(\mathrm{N}=7,39 \mathrm{wk})$, pre-term controls $(\mathrm{N}=6,29 \mathrm{wk})$, early-onset $\mathrm{PE}(\mathrm{N}=10,30 \mathrm{wk})$ and normotensive IUGR placentas $(\mathrm{N}=6,31.5 \mathrm{wk})$ from a cohort of samples previously included in our publications $[31,32]$. The selection criteria used for pre-eclampsia was the onset of new hypertension and proteinuria after 20 weeks of gestation; hypertension being defined as two or more recordings of a diastolic blood pressure of $90 \mathrm{mmHg}$ or more taken at least $4 \mathrm{~h}$ apart, proteinuria taken as the excretion of $300 \mathrm{mg}$ protein or more over a $24 \mathrm{~h}$ period. All preeclampsia cases were early-onset, defined as an onset between 20 and 34 weeks of gestation, and all were associated with IUGR. IUGR was defined according to established criteria [33], i.e. fetal biometry was below the 10th centile for gestational age according to local reference values, and umbilical artery Doppler flow velocity was abnormal (Doppler score class II and III). The pre-term control placentas were from women who had preterm but otherwise uneventful pregnancies, or late terminations of pregnancy for medical reasons. Fetal growth had been normal and birthweight was always above 10th percentile. The umbilical and uterine Doppler measurements were normal. The membranes were never ruptured for longer than $12 \mathrm{~h}$ and signs of chorioamnionitis were also excluded by histological studies. The cases included in this study all delivered by a caesarean section, and comprised of 2 late terminations of pregnancy (due to autosomal recessive polycystic kidneys or renal agenesis), 3 cases of early pre-mature rupture of membranes (no chorioamnionitis), and 1 case of early pre-term labour. However, preterm deliveries by definition are not normal controls. We therefore included an additional group of term controls from healthy uneventful pregnancies with normal umbilical and uterine artery Doppler waveforms. Placental tissue samples were snap-frozen immediately and stored at $-80^{\circ} \mathrm{C}$, and fixed in $4 \%$ PFA and processed for IHC. All placental samples were obtained from 3 to 4 placental regions, 
and several regions were incorporated into paraffin sections of the pathological placentas. However, these different samples were not pooled for Western blotting, for which a piece was cut from a block of frozen tissue.

\section{Culture of placental explants}

Villous samples were taken midway between the chorionic and basal plates, from the periphery of lobules free of visible infarction, calcification, haematoma or tears from term non-laboured placentas within $10 \mathrm{~min}$ of delivery, as previously described [34]. Following transport and tissue dissection, small placental explants ( $\sim 5 \mathrm{~mm}$ thick) were cultured under following conditions for $24 \mathrm{~h}$ : normoxia $\left(10 \% \mathrm{O}_{2}\right.$, $5 \% \mathrm{CO}_{2}$ ), hypoxia-reoxygenation ( $\mathrm{HR}$; hypoxia $\left(0.5 \% \mathrm{O}_{2} / 94.5 \% \mathrm{~N}_{2} / 5 \%\right.$ $\left.\mathrm{CO}_{2}\right)$ for $1 \mathrm{~h}$, subsequent reoxygenation at normoxia $\left(10 \% \mathrm{O}_{2} / 85 \% \mathrm{~N}_{2} /\right.$ $5 \% \mathrm{CO}_{2}$ ) for $24 \mathrm{~h}$ ), or $\mathrm{H}_{2} \mathrm{O}_{2}(1-1000 \mathrm{mM}$ for $24-48 \mathrm{~h})$.

\section{Western blotting}

We followed our previously described protocol [31]. Briefly, frozen tissue samples were homogenised in ice-cold lysis buffer and protein concentrations determined using a BCA protein assay kit (Sigma, Poole, UK). Lysates were mixed with $3 \mathrm{x}$ SDS PAGE sample buffer, boiled for $5 \mathrm{~min}$ and allowed to cool to room temperature. Equal amounts of protein $(30-50 \mu \mathrm{g})$ were separated by sodium dodecyl sulphate-polyacrylamide gel electrophoresis, using $7.5-12.5 \%$ polyacrylamide resolving gels, and transferred onto nitrocellulose membrane (Invitrogen, Paisley, UK), and subjected to immunoblot analysis. Membranes were blocked for $1 \mathrm{~h}$ at $25{ }^{\circ} \mathrm{C}$ in $5 \%$ milk diluted in Tris-buffered saline (TBS) and $0.1 \%$ Tween 20 and incubated with the following primary antibodies overnight at $4{ }^{\circ} \mathrm{C}$ : anti-p21 (Cell Signaling; \#2947), anti-p16 (Abcam; ab51243), or anti-cGAMP (Abcam; ab48508). After washing and incubating with secondary antibodies, immunoreactive proteins were visualized by the ECL plus chemiluminescence system following the manufacturer's instructions (Amersham Biosciences, Bucks., UK). Protein bands were quantified using Image $\mathrm{J}$ software (National Institutes of Health, http://rsb.info.nih.gov/ij/). Protein loading was normalized against Poncaeu S staining [35]. The values are expressed as a percentage of the control lysate $(100 \%)$ for each experiment.

\section{Immunostaining}

Immunohistochemistry was performed as previously described $[31,36]$. Briefly, following rehydration in xylene and graded ethanol, endogenous peroxidase was quenched with $\mathrm{H}_{2} \mathrm{O}_{2}$ and sections underwent heat antigen retrieval in Tris-EDTA buffer. The following antibodies were applied and incubated at 4 C overnight: p21 (Cell Signaling; \#2947), p16 (Abcam; ab51243), cGAMP (Abcam; ab224144), $\gamma$ H2AX (Cell Signaling; \#7631) and 8-hydroxy-2'-deoxy-guanosine (8OHdG; Abcam; ab48508). The next day, sections were incubated with biotin-labelled species specific antibodies, Vectastain Elite ABC kit (Vector Labs) and SigmaFast DAB (Sigma, Poole, UK).

Stained slides were scanned using a NanoZoomer scanner (Hamamatsu, Welwyn Garden City, UK). Each slide was visualized and several images (6-10 per placenta) were captured per placenta, with the observer blind to the study group. The number of p21 and 8OHdG positive trophoblast nuclei was quantified and expressed as a percentage of the total number of trophoblast nuclei counted. The staining of cGAMP expression in the trophoblast was determined semi-quantitatively using Image $\mathrm{J}$.

\section{Sudan-black-B staining for lysosomes}

Placental paraffin sections were dewaxed and brought to $70 \%$ ethanol. They were incubated in a saturated Sudan-Black-B solution (dissolved in $70 \%$ ethanol, freshly filtered before use) by adding a drop of SBB to a clean slide and inverting the section face down in order to prevent dye precipitation for about $8 \mathrm{~min}$. Sections were rinsed in $50 \%$ ethanol and placed in distilled water. Nuclear Fast Red stain $(0.1 \%$ solution, Sigma, UK) was subsequently applied for $2 \mathrm{~min}$, sections washed and mounted in a gelatin/glycerol/phenol aqueous mounting solution. Stained slides were scanned using a NanoZoomer scanner (Hamamatsu, Welwyn Garden City, UK). Each slide was visualized and several images were captured.

\section{Statistical analysis}

Data are expressed as mean \pm SD. Comparisons were made using a two-tailed Student's $t$-test where only two groups of samples were compared, or ANOVA with a Tukey's multiple comparison post-hoc test if more than two groups of samples were compared. Differences were considered to be significant at $P \leq 0.05$.

\section{Results}

Expression of senescence markers across gestation and in post-mature placentas

Significant increases in p21, p16 and cGAMP were observed in homogenates of healthy placentas with advancing gestational age (Fig. 1A), consistent with a report showing p16, p21, p53 and SA- $\beta$-gal in the syncytiotrophoblast at term [6]. Given the rise in p21 with gestational age, we quantified the number of p21-positive syncytiotrophoblast nuclei in an archival collection of post-mature placentas delivered 7-20 days following the due date [30], and observed a significant increase compared to term controls (5.7 vs. 1.3\%; Fig. 2A, C). Post-mature placentas also showed abundant staining with the senescence biomarker Sudan-Black-B, which localises to lipofuscin, an aggregate of oxidised proteins, lipids and metals (Fig. 2A). In addition, cGAMP was significantly increased in post-mature placentas, indicating increased cytoplasmic DNA in the syncytiotrophoblast (Fig. 2A, C). Abnormal nuclear appearances were observed in archival EM blocks of post-mature placentas, with dense heterochromatin being observed in some nuclear profiles but dissolution of the chromatin structure in others (Fig. 2B).

\section{Evidence of senescence in pathological placentas}

We compared p21, p16 and cGAMP in term and preterm control placentas with samples from early-onset PE (PE). Levels of p21 were lowest in term control placentas, and significantly higher in both preterm controls and PE (Fig. 1B). The increase in preterm controls may reflect the underlying placental pathology prompting their delivery. There were no significant differences in $\mathrm{p} 16$ or cGAMP protein among the groups (Fig. 1B).

Placentas from cases of PE suffer more severe malperfusion than those from IUGR alone [37], which may stimulate an exaggerated inflammatory response and contribute to the clinical syndrome [38]. Consistent with this hypothesis, levels of p21 were significantly higher in placentas from PE (Figs. 1C and 4A), but again there were no significant differences in p16 or cGAMP (Fig. 1C). Sudan-Black-B staining was strong in the PE and IUGR placentas, predominantly in the syncytiotrophoblast where discrete foci could be identified (Fig. 3). Immunostaining revealed nuclear syncytial localisation of p21 (Fig. 4A) and modified histone, $\gamma \mathrm{H} 2 \mathrm{AX}$ (Fig. 4B) in the pathological placentas, providing evidence for DDR pathway activation in these placentas.

Oxidative stress challenges can induce senescence and DNA damage in term placental explants in vitro

Acute challenge ( $24 \mathrm{~h}$ ) with HR induced similar changes in placental explants in vitro (Figs. 5-6), with significant increases in the 

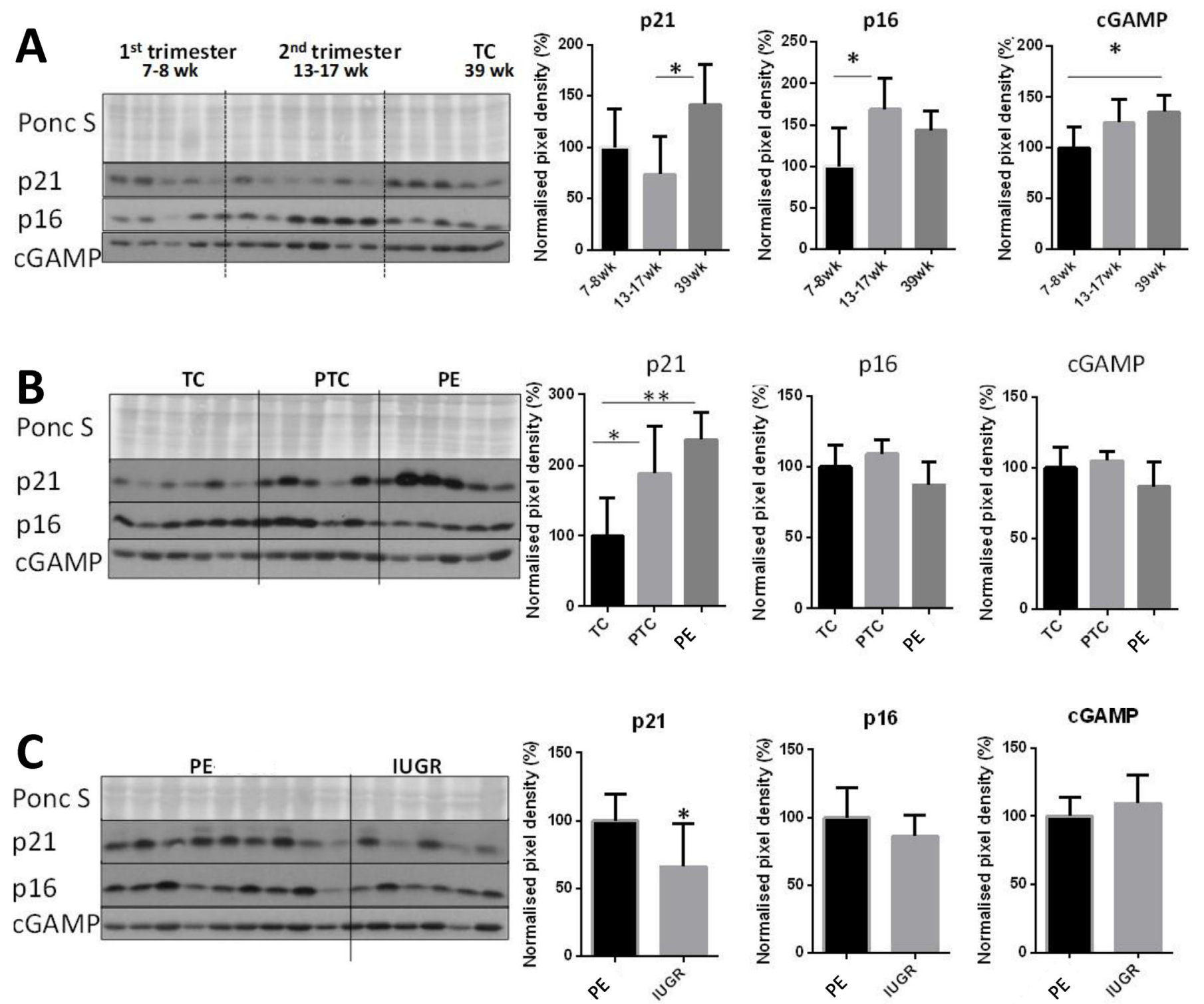

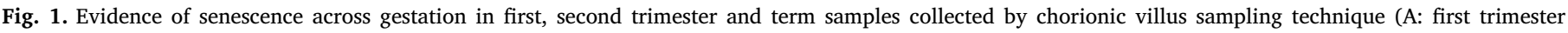

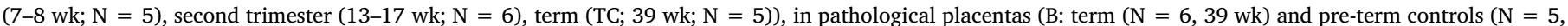

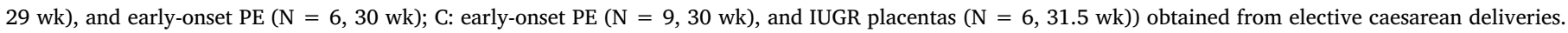

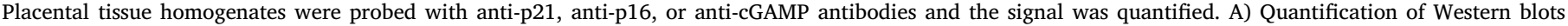

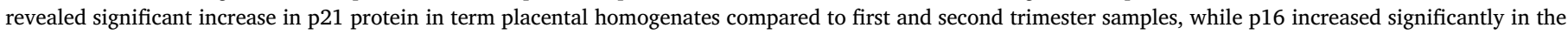

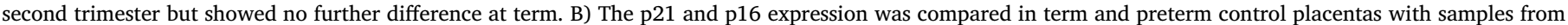

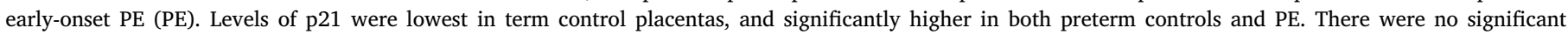

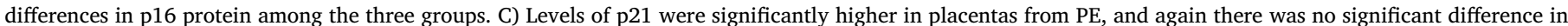

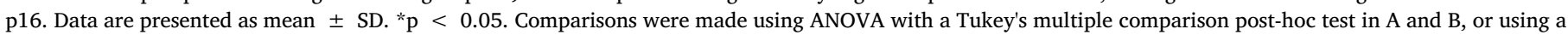
two-tailed Student's $t$-test where only two groups of samples were compared (C).

aggregation of lipofuscin, as detected by SBB (Fig. 5A), and significant increases in p21 (Figs. 5B and 6A) and p16 (Fig. 6A), and increased nuclear foci of $\gamma \mathrm{H} 2 \mathrm{AX}$ (Fig. 5C) compared to normoxic controls.

Oxidative stress causes DNA damage, and we examined immunoreactivity for 8-hydroxy-2'-deoxy-guanosine (8-OHdG), a marker of oxidised DNA (Fig. 6B-D). The percentage of immunopositive nuclei was increased in pathological placentas compared to age-matched controls, with no differences between PE vs. IUGR (Fig. 6B). These changes could be mimicked in vitro in term placental explants challenged with $\mathrm{H}_{2} \mathrm{O}_{2}(0-1 \mathrm{M})$ for 24-48 h (Fig. 6C-D).

\section{Discussion}

This study provides evidence of senescence in normal placentas across gestation, in post-mature placentas and in pathological pregnancies. We report significantly increased levels of p21, p16 and cGAMP in homogenates of healthy placentas with gestational age. This is consistent with a report showing p16, p21, p53 and SA- $\beta$-gal in term syncytiotrophoblast [6]. Londero et al. [29] found increased levels of 8OHdG, APE1 and p53 with gestational age, but a reduction in p21. This difference may reflect the method of collection of early pregnancy samples. Our 7-17 week tissues were collected using chorionic villus sampling that avoids stress induced by suction curettage [39] that may raise levels artificially ex vivo. Placental ageing thus seems to be a 
A

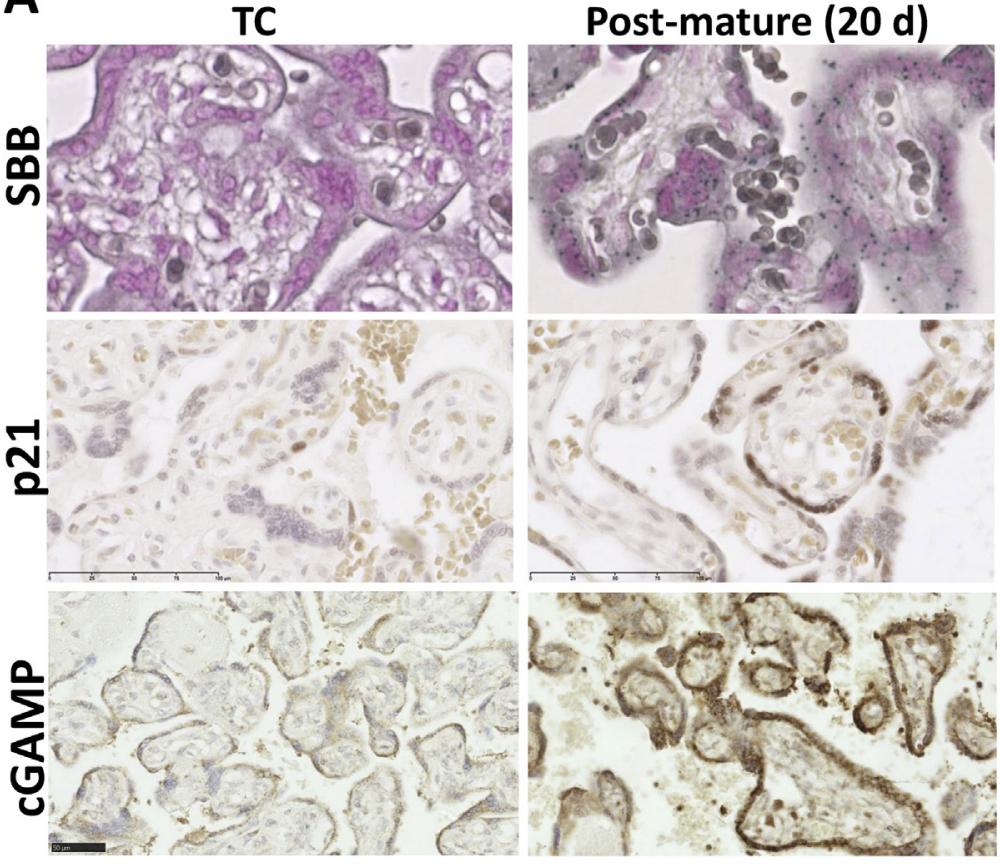

B

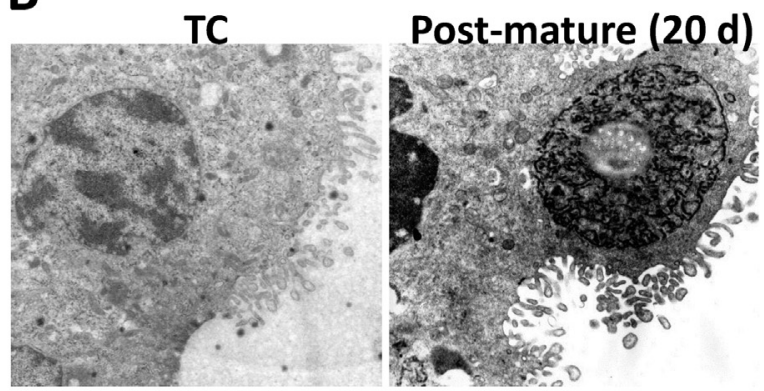

C

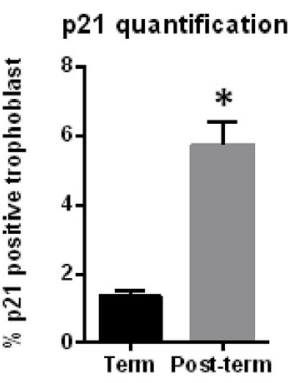

cGAMP quantification

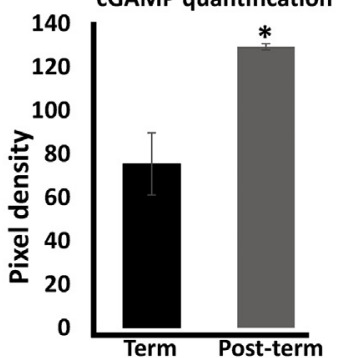

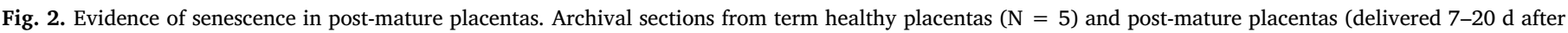

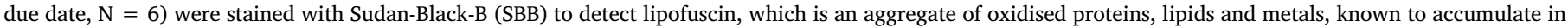

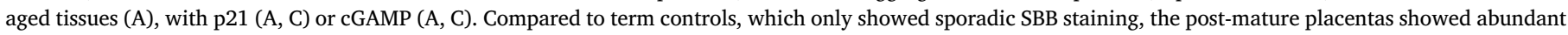

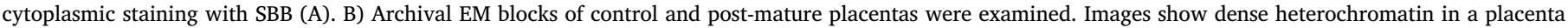

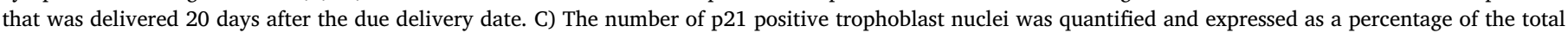

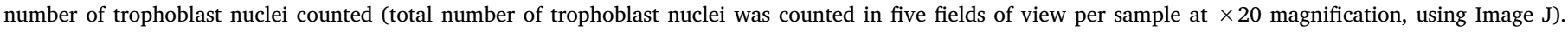

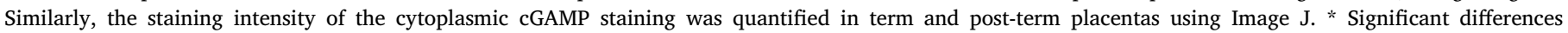
$(\mathrm{P}<0.05, t$-test $)$.

regular response, and this physiological phenomenon was first described in the 1970s [18-20]. As pregnancy advances, placental cells undergo morphological changes, such as nuclear pyknosis [18], fibrinoid accumulation [19], and oxidative modification of nucleic acids [20], indicative of senescence.

Given the evidence of ageing in healthy placentas across gestation, we analysed a collection of archival paraffin and EM sections of postmature placentas from patients who delivered 14-20 days after their due delivery dates in the 1970s. It is now recognised that the risk of fetal demise increases with gestational age in term patients, as there is a significant increase in the rate of stillbirth commencing at $36-37 \mathrm{wk}$ gestation [22,23]. Consequently, such severe post-maturity is rarely seen in modern obstetric practice for delivery is usually induced after $41 \mathrm{wk}$. These severely post-mature placentas showed significant staining of the syncytiotrophoblast with Sudan-Black-B, a marker for aggregates of oxidised lipids, proteins and metals referred to as lipofuscin in ageing cells. SBB staining was largely absent in healthy term placentas. The post-mature placentas also showed increased nuclear
SBB

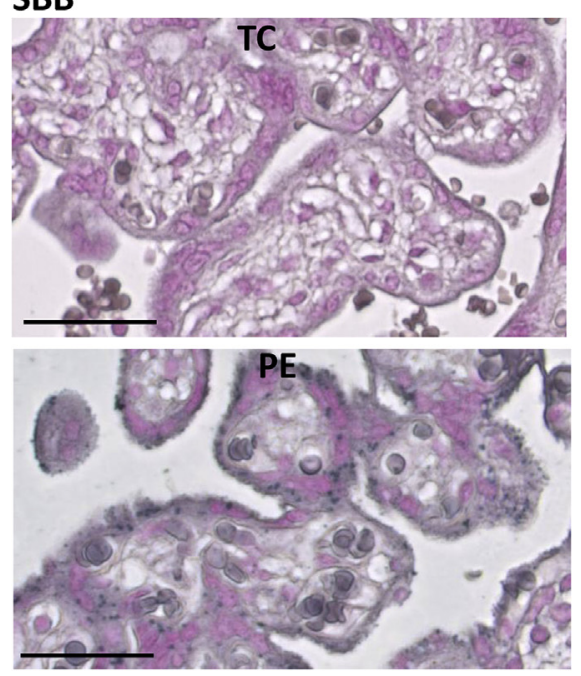

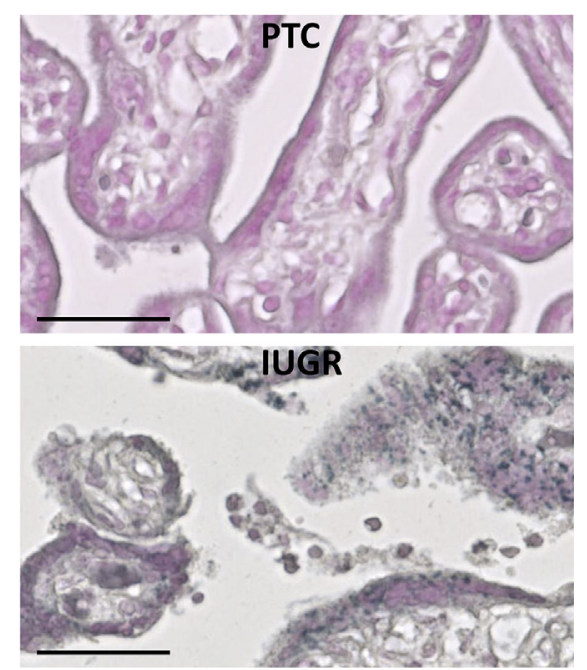

Fig. 3. Staining with Sudan-Black-B (SBB) to detect lipofuscin, an aggregate of oxidised proteins, lipids and metals, in control and pathological placentas. Immunostaining revealed abundant nuclear localisation in the syncytiotrophoblast of the pathological placentas. 
A

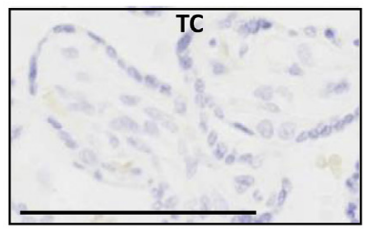

B

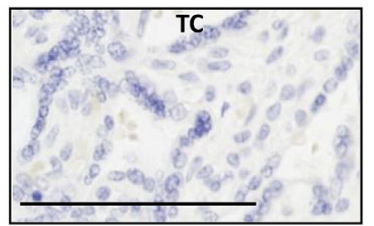

p21
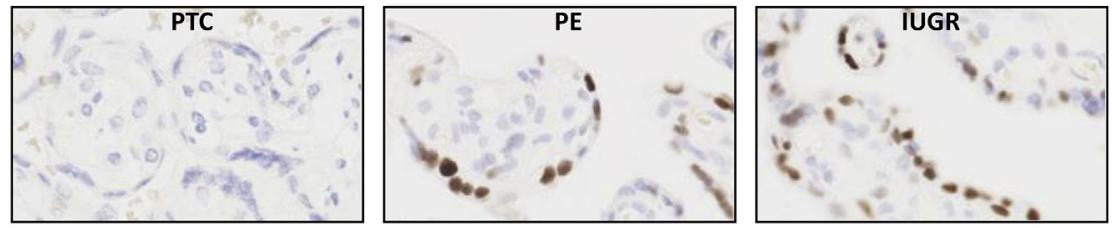

YH2AX
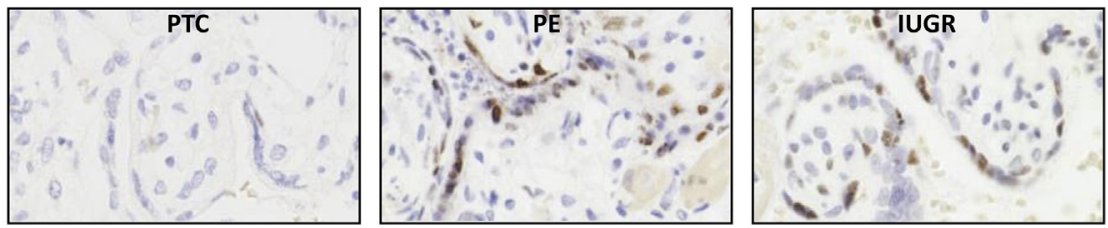

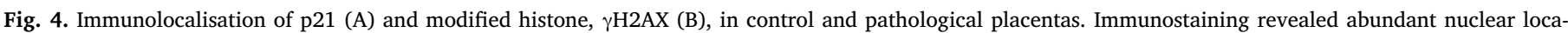
lisation of p21 (A) and modified histone $\gamma \mathrm{H} 2 \mathrm{AX}(\mathrm{B})$ in the syncytiotrophoblast of the pathological placentas.

abundance of p21 and staining intensity for cGAMP in the syncytiotrophoblast. The latter is indicative of increased cytoplasmic DNA, consistent with the abnormal nuclear appearances indicative of dissolution of the normal chromatin structure and potential release of DNA. Hence, our data show a striking increase in the incidence of senescence in the post-mature placentas (41-44 wk), compared to term controls (39-40 wk), validating the concept of physiological trophoblast ageing. Our results are in agreement with those of Maiti et al. who studied term placentas ( $39 \mathrm{wk}$ ), late term placentas $(>41 \mathrm{wk}$ ) and placentas from unexplained stillbirth. These authors reported increased aldehyde oxidase 1 expression, increased oxidation of DNA/RNA (8OHdG) and lipids, perinuclear location of lysosomes, and larger autophagosomes in both later term and stillborn placentas, compared to placentas from term deliveries [21]. Overall, these findings suggest that premature ageing of the placenta could account for the placental dysfunction that characterizes some cases of stillbirth.

We next analysed evidence of senescence in pathological samples from early-onset PE (PE) and found significant increases in SudanBlack-B staining, syncytiotrophoblast nuclear expression of p21 and nuclear foci containing modified chromatin $(\gamma \mathrm{H} 2 \mathrm{AX})$ compared to term and pre-term controls. There were no significant differences in the levels of p16 or cGAMP among the groups. Placentas from cases of PE suffer more severe malperfusion than those from IUGR alone [37], which may stimulate an exaggerated inflammatory response that contributes to the clinical syndrome of PE [38]. Consistent with this hypothesis, levels of $\mathrm{p} 21$ were significantly higher in placentas from PE than in IUGR alone, but again there were no significant differences in p16 or cGAMP. Our data are supported by previous reports of telomere shortening in placentas from IUGR and PE pregnancies [28].

Hypoxia-reoxygenation is a potent inducer of oxidative stress, inflammation and apoptosis in term placental explants [34,40]. In this study, the HR challenge also induced senescent changes in term placental explants, which displayed significant increases in lipofuscin staining, p21 and p16, and nuclear foci of modified histone $\gamma \mathrm{H} 2 \mathrm{AX}$,

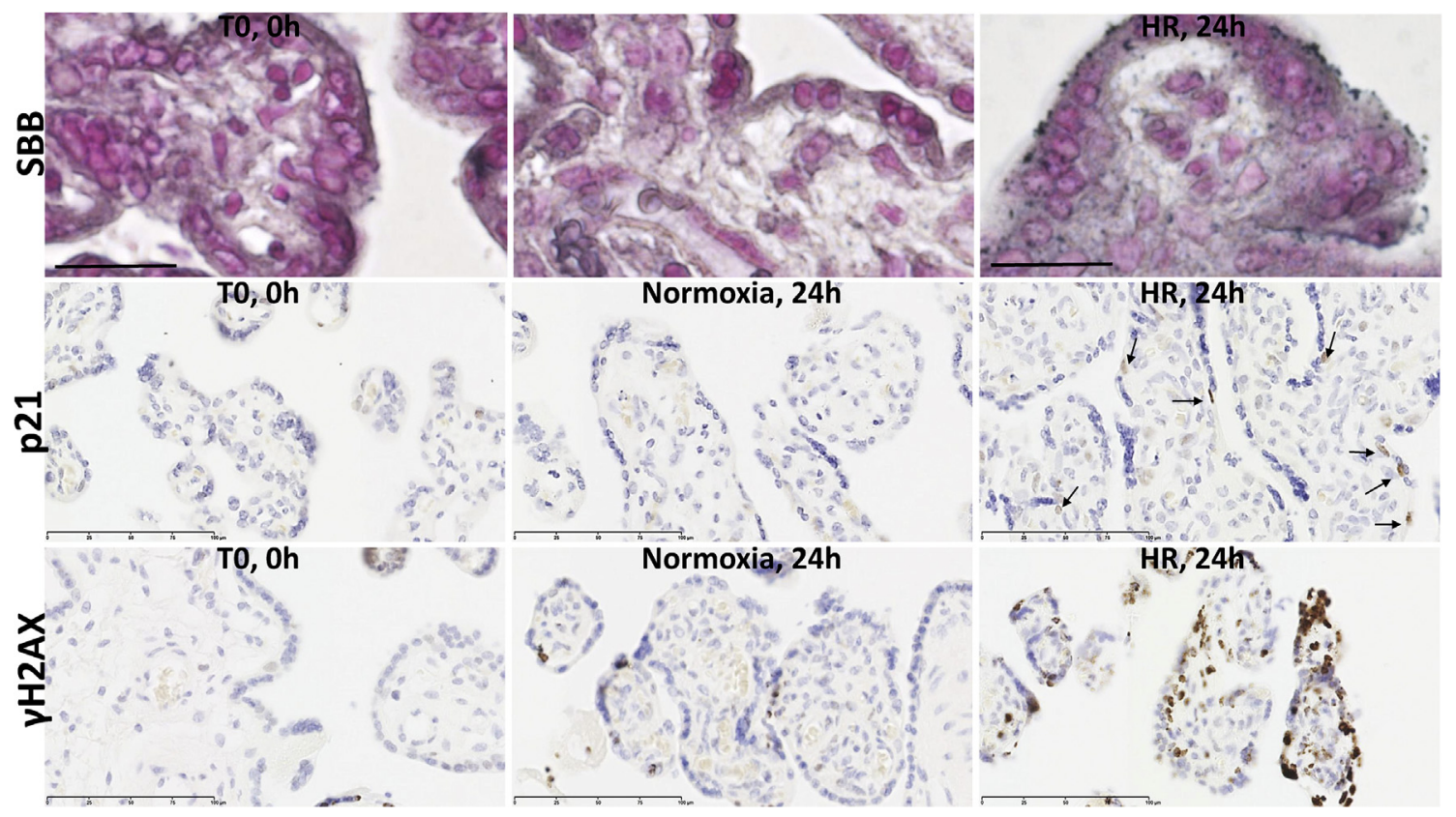

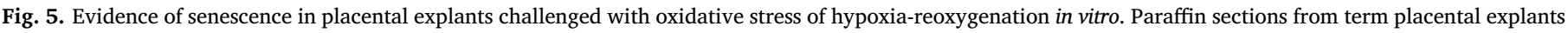

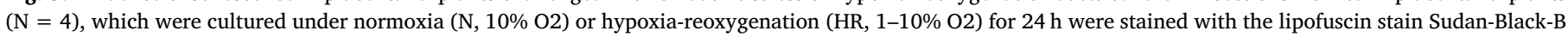

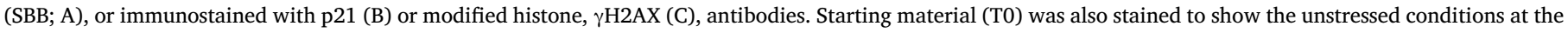
start of the culture. 

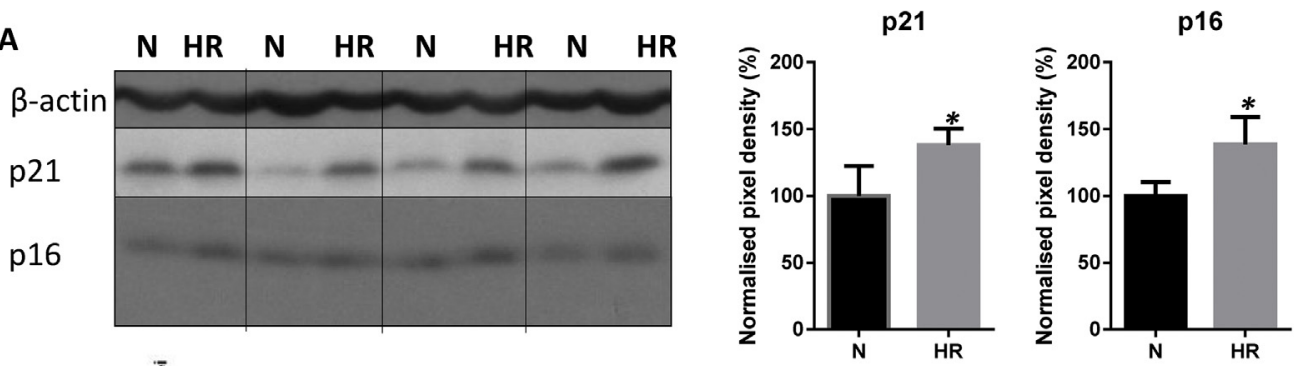

B

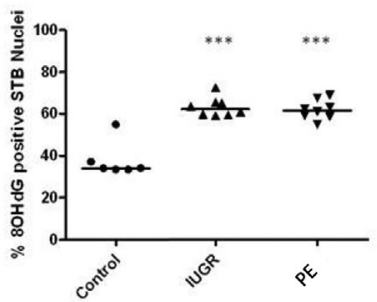

D
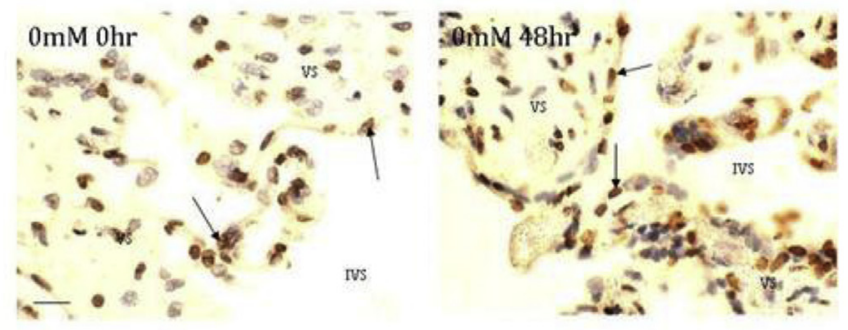

C
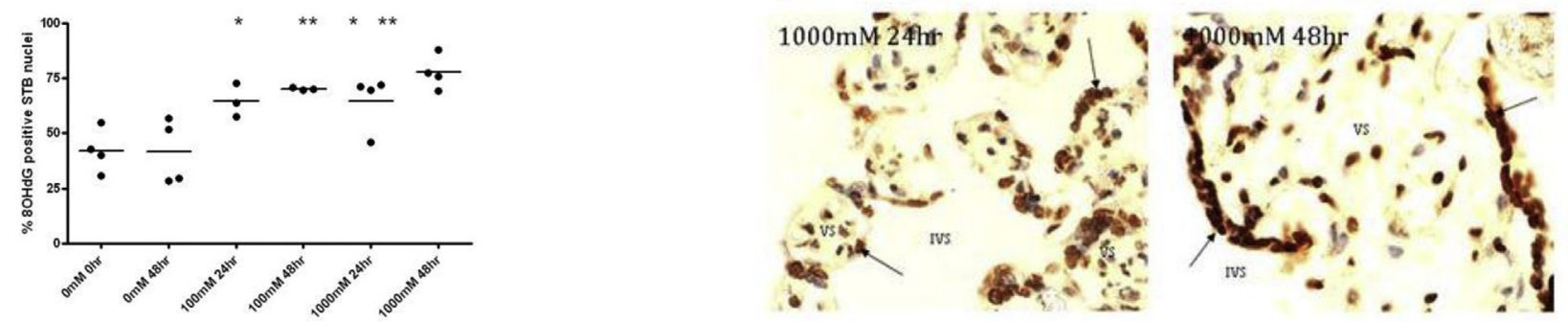

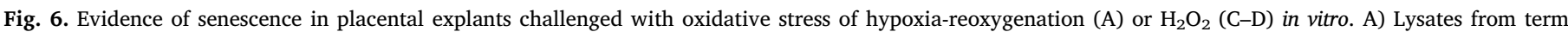

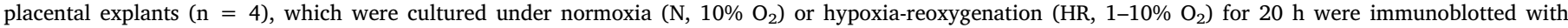

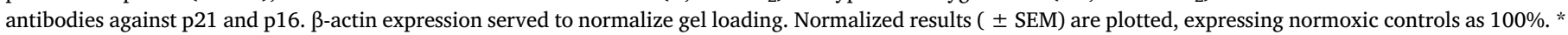

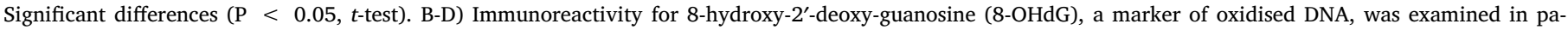

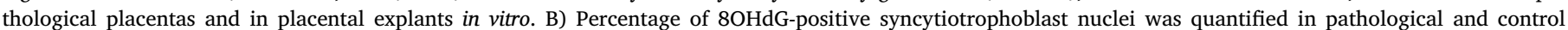

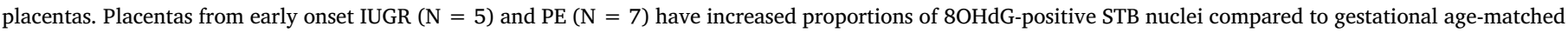

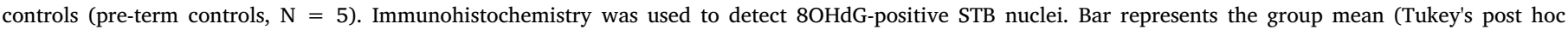

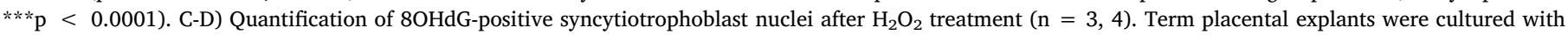

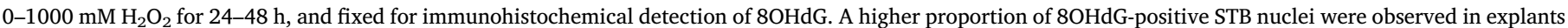

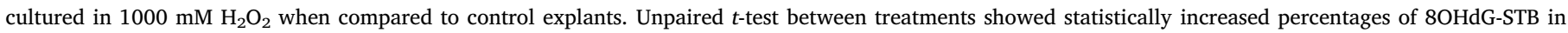

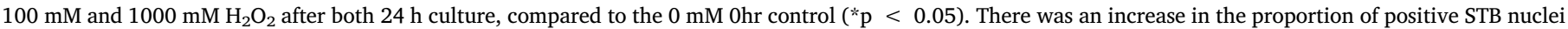

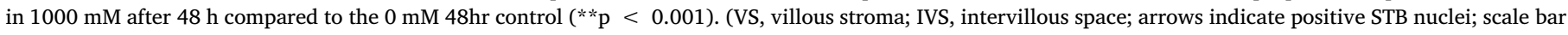
$20 \mu \mathrm{m})$.

compared to normoxic controls. In addition, our in vitro experiments show that oxidative stress causes DNA damage. Compared to cultures under normoxia, treatment of term placental explants with $\mathrm{H}_{2} \mathrm{O}_{2}$ for 24 or $48 \mathrm{~h}$ induced an increased percentage of nuclei immunopositive for 8-hydroxy-2'-deoxy-guanosine (8-OHdG), a marker of oxidised DNA/ RNA. Similar changes were observed in pathological placentas, consistent with the hypothesis that oxidative stress is the main inducer of senescence in vivo.

In conclusion, we show increasing levels of senescence in normal placentas with gestational age, and in pathological placentas. Oxidative stress triggers these changes in placental explants, and may be the precipitating insult in vivo. In severe cases, the consequent pro-inflammatory senescence-associated secretory phenotype may contribute to the pathophysiology of early-onset pre-eclampsia, for many of the cytokines released, such as IL-1, IL-6, IL-8, are common to both conditions.

\section{Conflict of interest}

The authors have no conflict of interest to declare.

\section{Funding sources}

Supported by the Wellcome Trust (084804/2/08/Z).

\section{Acknowledgements}

We thank the Cambridge Comprehensive Biomedical Research Centre and the staff of the Rosie Hospital for their help in collecting the normal term placentas. The study was funded by the Wellcome Trust (084804/2/08/Z) and an Anatomical Society Studentship (NF).

\section{References}

[1] J. Bartek, J. Bartkova, J. Lukas, DNA damage signalling guards against activated oncogenes and tumour progression, Oncogene 26 (56) (2007) 7773-7779.

[2] J. Krishnamurthy, C. Torrice, M.R. Ramsey, G.I. Kovalev, K. Al-Regaiey, L. Su, N.E. Sharpless, Ink4a/Arf expression is a biomarker of aging, JCI (J. Clin. Investig.) 114 (9) (2004) 1299-1307.

[3] C. Wang, D. Jurk, M. Maddick, G. Nelson, C. Martin-Ruiz, T. von Zglinicki, DNA damage response and cellular senescence in tissues of aging mice, Aging Cell 8 (3) (2009) 311-323.

[4] T. Minamino, I. Komuro, Vascular cell senescence: contribution to atherosclerosis, Circ. Res. 100 (1) (2007) 15-26.

[5] R. Bhat, E.P. Crowe, A. Bitto, M. Moh, C.D. Katsetos, F.U. Garcia, F.B. Johnson, J.Q. Trojanowski, C. Sell, C. Torres, Astrocyte senescence as a component of Alzheimer's disease, PLoS One 7 (9) (2012) e45069. 
[6] A. Chuprin, H. Gal, T. Biron-Shental, A. Biran, A. Amiel, S. Rozenblatt, V. Krizhanovsky, Cell fusion induced by ERVWE1 or measles virus causes cellular senescence, Genes Dev. 27 (21) (2013) 2356-2366.

[7] M. Collado, M. Serrano, Senescence in tumours: evidence from mice and humans, Nature reviews 10 (1) (2010) 51-57.

[8] J.M. van Deursen, The role of senescent cells in ageing, Nature 509 (7501) (2014) $439-446$.

[9] M. Narita, M. Narita, V. Krizhanovsky, S. Nunez, A. Chicas, S.A. Hearn, M.P. Myers, S.W. Lowe, A novel role for high-mobility group a proteins in cellular senescence and heterochromatin formation, Cell 126 (3) (2006) 503-514.

[10] R. Zhang, W. Chen, P.D. Adams, Molecular dissection of formation of senescenceassociated heterochromatin foci, Mol. Cell Biol. 27 (6) (2007) 2343-2358.

[11] F. Rodier, J.P. Coppe, C.K. Patil, W.A. Hoeijmakers, D.P. Munoz, S.R. Raza, A. Freund, E. Campeau, A.R. Davalos, J. Campisi, Persistent DNA damage signalling triggers senescence-associated inflammatory cytokine secretion, Nat. Cell Biol. 11 (8) (2009) 973-979.

[12] J.P. Coppe, C.K. Patil, F. Rodier, Y. Sun, D.P. Munoz, J. Goldstein, P.S. Nelson, P.Y. Desprez, J. Campisi, Senescence-associated secretory phenotypes reveal cellnonautonomous functions of oncogenic RAS and the p53 tumor suppressor, PLoS Biology 6 (12) (2008) 2853-2868.

[13] J.F. Passos, G. Nelson, C. Wang, T. Richter, C. Simillion, C.J. Proctor, S. Miwa, S. Olijslagers, J. Hallinan, A. Wipat, G. Saretzki, K.L. Rudolph, T.B. Kirkwood, T. von Zglinicki, Feedback between p21 and reactive oxygen production is necessary for cell senescence, Mol. Syst. Biol. 6 (2010) 347.

[14] J. Campisi, Cellular senescence: putting the paradoxes in perspective, Curr. Opin. Genet. Dev. 21 (1) (2011) 107-112.

[15] E.A. Georgakopoulou, K. Tsimaratou, K. Evangelou, P.J. Fernandez Marcos, V. Zoumpourlis, I.P. Trougakos, D. Kletsas, J. Bartek, M. Serrano, V.G. Gorgoulis, Specific lipofuscin staining as a novel biomarker to detect replicative and stressinduced senescence, A method applicable in cryo-preserved and archival tissues, Aging (Albany NY) 5 (1) (2013) 37-50.

[16] Z. Dou, K. Ghosh, M.G. Vizioli, J. Zhu, P. Sen, K.J. Wangensteen, J. Simithy, Y. Lan, Y. Lin, Z. Zhou, B.C. Capell, C. Xu, M. Xu, J.E. Kieckhaefer, T. Jiang, M. ShoshkesCarmel, K. Tanim, G.N. Barber, J.T. Seykora, S.E. Millar, K.H. Kaestner, B.A. Garcia, P.D. Adams, S.L. Berger, Cytoplasmic chromatin triggers inflammation in senescence and cancer, Nature 550 (7676) (2017) 402-406.

[17] S. Gluck, B. Guey, M.F. Gulen, K. Wolter, T.W. Kang, N.A. Schmacke, A. Bridgeman, J. Rehwinkel, L. Zender, A. Ablasser, Innate immune sensing of cytosolic chromatin fragments through cGAS promotes senescence, Nat. Cell Biol. 19 (9) (2017) 1061-1070.

[18] B.J. Martin, S.S. Spicer, Ultrastructural features of cellular maturation and aging in human trophoblast, J Ultrastruct Res 43 (1) (1973) 133-149.

[19] R. Burstein, S. Frankel, S.D. Soule, H.T. Blumenthal, Aging of the placenta: autoimmune theory of senescence, Am. J. Obstet. Gynecol. 116 (2) (1973) 271-276.

[20] P. Rosso, Placenta as an aging organ, Curr. Concepts Nutr. 4 (1976) 23-41.

[21] K. Maiti, Z. Sultana, R.J. Aitken, J. Morris, F. Park, B. Andrew, S.C. Riley, R. Smith, Evidence that fetal death is associated with placental aging, Am. J. Obstet. Gynecol. 217 (4) (2017) 441 e1-441 e14.

[22] J.F. Froen, M. Arnestad, K. Frey, A. Vege, O.D. Saugstad, B. Stray-Pedersen, Risk factors for sudden intrauterine unexplained death: epidemiologic characteristics of singleton cases in Oslo, Norway, 1986-1995, Am. J. Obstet. Gynecol. 184 (4) (2001) 694-702.

[23] E.A. Nohr, B.H. Bech, M.J. Davies, M. Frydenberg, T.B. Henriksen, J. Olsen, Prepregnancy obesity and fetal death: a study within the Danish National Birth
Cohort, Obstet. Gynecol. 106 (2) (2005) 250-259.

[24] C.W.G. Redman, I.L. Sargent, Placental debris, oxidative stress and pre-eclampsia, Placenta 21 (2000) 597-602.

[25] C.A. Hubel, Oxidative stress in the pathogenesis of preeclampsia, Proc Soc Exp Biol Med 222 (3) (1999) 222-235.

[26] G.J. Burton, E. Jauniaux, Placental oxidative stress: from miscarriage to preeclampsia, J. Soc. Gynecol. Invest. 11 (2004) 342-352.

[27] J.J. Brosens, R. Pijnenborg, I.A. Brosens, The myometrial junctional zone spiral arteries in normal and abnormal pregnancies: a review of the literature, Am. J. Obstet. Gynecol. 187 (2002) 1416-1423.

[28] T. Biron-Shental, R. Sukenik-Halevy, Y. Sharon, L. Goldberg-Bittman, D. Kidron, M.D. Fejgin, A. Amiel, Short telomeres may play a role in placental dysfunction in preeclampsia and intrauterine growth restriction, Am. J. Obstet. Gynecol. 202 (4) (2010) e1-7 381.

[29] A.P. Londero, M. Orsaria, S. Marzinotto, T. Grassi, A. Fruscalzo, A. Calcagno, S. Bertozzi, N. Nardini, E. Stella, R.J. Lelle, L. Driul, G. Tell, L. Mariuzzi, Placental aging and oxidation damage in a tissue micro-array model: an immunohistochemistry study, Histochem. Cell Biol. 146 (2) (2016) 191-204.

[30] C. Jones, PhD Thesis, (1976).

[31] L. Lu, J. Kingdom, G.J. Burton, T. Cindrova-Davies, Placental stem villus arteria remodeling associated with reduced hydrogen sulfide synthesis contributes to human fetal growth restriction, Am. J. Pathol. 187 (4) (2017) 908-920.

[32] T. Cindrova-Davies, E.A. Herrera, Y. Niu, J. Kingdom, D.A. Giussani, G.J. Burton, Reduced cystathionine gamma-lyase and increased miR-21 expression are associated with increased vascular resistance in growth-restricted pregnancies: hydrogen sulfide as a placental vasodilator, Am. J. Pathol. 182 (4) (2013) 1448-1458.

[33] A. Lausman, F.P. McCarthy, M. Walker, J. Kingdom, Screening, diagnosis, and management of intrauterine growth restriction, J. Obstet. Gynaecol. Can. 34 (1) (2012) 17-28.

[34] T. Cindrova-Davies, O. Spasic-Boskovic, E. Jauniaux, D.S. Charnock-Jones, G.J. Burton, Nuclear factor-kappa B, p38, and stress-activated protein kinase mitogen-activated protein kinase signaling pathways regulate proinflammatory cytokines and apoptosis in human placental explants in response to oxidative stress: effects of antioxidant vitamins, Am. J. Pathol. 170 (5) (2007) 1511-1520.

[35] I. Romero-Calvo, B. Ocon, P. Martinez-Moya, M.D. Suarez, A. Zarzuelo, O. Martinez Augustin, F.S. de Medina, Reversible Ponceau staining as a loading control alternative to actin in Western blots, Anal. Biochem. 401 (2) (2010) 318-320.

[36] T. Cindrova-Davies, H.W. Yung, J. Johns, O. Spasic-Boskovic, S. Korolchuk, E. Jauniaux, G.J. Burton, D.S. Charnock-Jones, Oxidative stress, gene expression, and protein changes induced in the human placenta during labor, Am. J. Pathol 171 (4) (2007) 1168-1179.

[37] I. Brosens, R. Pijnenborg, L. Vercruysse, R. Romero, The "Great Obstetrical Syndromes" are associated with disorders of deep placentation, Am. J. Obstet. Gynecol. 204 (3) (2011) 193-201.

[38] C.W. Redman, G.P. Sacks, I.L. Sargent, Preeclampsia: an excessive maternal inflammatory response to pregnancy, Am. J. Obstet. Gynecol. 180 (2 Pt 1) (1999) 499-506.

[39] T. Cindrova-Davies, M.T. van Patot, L. Gardner, E. Jauniaux, G.J. Burton, D.S. Charnock-Jones, Energy status and HIF signalling in chorionic villi show no evidence of hypoxic stress during human early placental development, Mol. Hum. Reprod. 21 (3) (2015) 296-308.

[40] T.-H. Hung, J.N. Skepper, D.S. Charnock-Jones, G.J. Burton, Hypoxia-reoxygenation: a potent inducer of apoptotic changes in the human placenta and possible etiological factor in preeclampsia, Circ. Res. 28 (2002) 1274-1281. 